\title{
Eosinophilic colitis as a complication of the hypereosinophilic syndrome
}

\author{
A.M. Shah* and M. Joglekar $\dagger$ \\ Department of Medicine, Bridgend General Hospital, Quarella Road, Bridgend, Mid Glam., UK.
}

Summary: A patient with the hypereosinophilic syndrome complicated by a severe eosinophilic colitis is reported. The association has not previously been recorded.

\section{Introduction}

The hypereosinophilic syndrome (HES) is characterized by persistent peripheral blood eosinophilia and a range of multi-system complications. A severe eosinophilic colitis has not been previously described in association with this syndrome. We report a patient with HES and eosinophilic colitis who was successfully treated with hydroxyurea.

\section{Case report}

A previously healthy 20 year old Caucasian housewife presented in 1980 with a 3-week history of nonproductive cough, dyspnoea and wheezing. She smoked 30 cigarettes a day. There was no history of allergy or of recent travel abroad. Examination revealed fever, tachycardia and a blood pressure of $120 / 80 \mathrm{~mm} \mathrm{Hg}$. There were widespread expiratory wheezes in the chest. The peak expiratory flow rate (PFR) was 260 litres/min, improving to 300 litres/min after inhaled salbutamol. The peripheral white cell count (WBC) was $28 \times 10^{9} / 1$ (eosinophils $11.2 \times 10^{9}$ / 1), haemoglobin concentration $13.5 \mathrm{~g} / \mathrm{dl}$. Blood urea, electrolytes and liver function tests were normal. The chest X-ray showed diffuse bilateral pulmonary infiltrates. Blood and sputum cultures and serological tests for viruses, hydatid disease, amoebiasis and toxocara were negative.

There were no ova, cysts or parasites in the faeces. No auto-antibodies were detected. Aspergillus precipitins and skin tests were negative. Initial therapy with erythromycin and bronchodilators produced

Correspondence: A.M. Shah, M.B., M.R.C.P., 2 Morlais Street, Roath, Cardiff CF2 5HQ, UK.

*Present address: University Hospital of Wales, Heath Park, Cardiff, UK.

†Present address: Caerphilly Miners' Hospital, Caerphilly, Mid Glam., UK.

Accepted: 16 December 1986 some clinical improvement but the radiological appearances and eosinophilia persisted until prednisolone $40 \mathrm{mg} /$ day was added.

A diagnosis of pulmonary eosinophilia with asthma was made. The patient was discharged on inhaled corticosteroids and bronchodilators. Recurrent episodes of wheezing, low PFR and pulmonary infiltrates over the next 3 years responded to short courses of oral prednisolone.

In September 1983 she presented with a 3-week history of bloody diarrhoea with 5-6 loose motions per day. There was no abdominal pain or weight loss. She was taking prednisolone $5 \mathrm{mg} /$ day. There was increased wheezing, and ptosis of the right eye. Abdominal and rectal examination was normal. The haemoglobin concentration was $13.0 \mathrm{~g} / \mathrm{dl}$, WBC $15 \times 10^{9} / 1$ (eosinophils $2.7 \times 10^{9} / 1$ ). The erythrocyte sedimentation rate (ESR) was $20 \mathrm{~mm}$ in the first hour. Stool cultures and microscopy were normal. Flexible sigmoidoscopy to the mid-descending colon revealed widespread inflammation with ulceration, contact bleeding and mucus discharge. Multiple colonic and rectal biopsies were taken. These demonstrated a mild attenuation of the surface epithelium, and a moderate chronic inflammatory infiltrate strikingly rich in eosinophils (Figure 1). There was no evidence of vasculitis or granuloma formation. No parasites were detected. A barium enema was normal. Prednisolone $30 \mathrm{mg} /$ day and hydrocortisone enemata induced remission of all symptoms. She was then maintained on 5-10 mg prednisolone per day.

The patient was readmitted as an emergency in July 1984. She had been unwell for one week with confusion, myalgia and poor balance. Wheezing and bloody diarrhoea had recurred. She was taking prednisolone $5 \mathrm{mg} / \mathrm{day}$. On examination she appeared ill, unsteady on her feet, confused and disorientated. There was a mild pyrexia and bilateral finger nail splinter haemorrhages. The pulse was 110 /minute, 


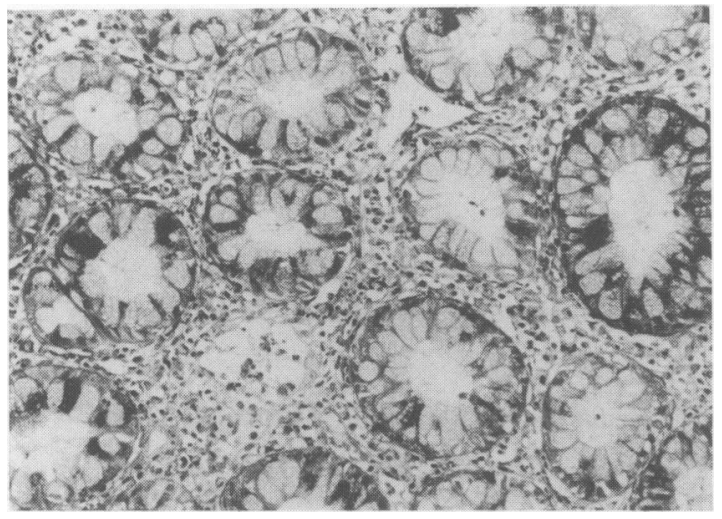

Figure 1 Colonic mucosa with clusters of eosinophils between the glands (Giemsa $\times 66$, green filter)

blood pressure $100 / 60 \mathrm{~mm} \mathrm{Hg}$. The cranial nerves were intact. Muscle power was reduced in the right arm with hypertonia and hyper-reflexia. There were no sensory signs. Auscultation of the heart revealed a gallop rhythm. The jugular venous pressure was normal and the lung bases clear. Abdominal examination was normal.

The WBC had risen to $35 \times 10^{9} / 1$ (eosinophils $19.95 \times 10^{9} / 1$ ), haemoglobin concentration $14.5 \mathrm{~g} / \mathrm{dl}$. The ESR was $21 \mathrm{~mm} /$ hour. Blood urea, electrolytes and glucose were normal. The aspartate transaminase was $69 \mathrm{IU} / 1$ (normal $0-40 \mathrm{IU} / 1$ ) and the lactate dehydrogenase $610 \mathrm{IU} / 1$ (normal 0-250 IU/1). Blood, cerebrospinal fluid and stool cultures were all sterile. The chest X-ray showed bilateral diffuse infiltrates. The electrocardiogram showed diminished anterior $\mathbf{R}$ wave amplitude but no $T$ wave changes, and an echocardiogram was normal. Bone marrow aspiration revealed marked eosinophilia but no primitive cell preponderance. The serum IgE concentration was $341 \mathrm{kU} / 1$ (adult referencé range $<81 \mathrm{kU} / \mathrm{l}$ ).

A diagnosis of the hypereosinophilic syndrome was made. The prednisolone dosage was increased to $40 \mathrm{mg} /$ day and hydroxyurea $(2 \mathrm{~g} /$ day $)$ commenced 3 days later. There was a dramatic improvement in her symptoms and signs within one week. The eosinophil count dropped to $1.1 \times 10^{9} / 1$. Complete recovery occurred over the next 4 weeks and prednisolone was tailed off during this period. She was maintained on hydroxyurea ( $2 \mathrm{~g} /$ day) alone. Repeat flexible sigmoidoscopy in November 1984 revealed normal macroscopic appearances and colonic biopsies showed no eosinophilia or inflammation. Further mild episodes of bloody diarrhoea have responded to topical steroids, and the patient remains well on maintenance treatment with hydroxyurea $(1 \mathrm{~g} /$ day $)$ and inhaled corticosteroids 21 months later.

\section{Discussion}

The term hypereosinophilic syndrome (HES) was introduced by Hardy \& Anderson in $1968^{\prime}$ to describe patients with peripheral blood eosinophilia for which no cause could be found. Chusid et al. ${ }^{2}$ defined the syndrome more precisely and restricted the diagnosis to patients with (i) persistent eosinophilia $>1.5 \times 10^{9} / 1$, (ii) lack of evidence for any known cause and (iii) evidence of multi-organ disease.

The main clinical complications of HES are endomyocardial disease, skin lesions, thromboembolism, gastrointestinal symptoms, cerebral lesions, splenomegaly and respiratory disease. ${ }^{2,3}$ Diarrhoea is reported to occur in over one fifth of patients with HES and eosinophil infiltrates are occasionally described. $^{2,3}$ A severe eosinophilic colitis has not been previously described in association with HES.

Eosinophilic infiltration of the gastrointestinal tract occurs in many conditions including eosinophilic gastroenteritis, ulcerative colitis, allergic granulomatosis with angiitis (Churg-Strauss syndrome), helminthic disease, lymphoma and gastric cancer. ${ }^{4-6}$ An eosinophilic colitis with bloody diarrhoea and peripheral blood eosinophilia may occur in infancy and is suggested to be due to cows' milk intolerance. ${ }^{7}$ Eosinophilic gastroenteritis occurs in older childrepg and adults and usually presents with vomiting, diarrs hoea, weight loss and malabsorption. ${ }^{6}$ Infiltration with eosinophils is usually restricted to the stomach and small bowel, colonic disease being rare. ${ }^{6}$ The Churg-Strauss syndrome (CSS) may sometimes be difficult to distinguish from HES on clinical grounds. The major histological criteria for diagnosis of CSS are necrotizing vasculitis, extravascular granulomas and tissue infiltration by eosinophils. ${ }^{8}$ Long standing pulmonary involvement, vasculitic skin rashes and mononeuritis multiplex are often prominent features and the ESR is considerably raised during the vasculitic phase. ' The eosinophilia in CSS is usually of lesser magnitude than that in HES and easier to abolish with steroids. ${ }^{9}$

Our patient presented with respiratory symptoms, then developed an eosinophilic colitis and ptosis, and finally had a severe relapse with splinter haemorrhages, cardiac, respiratory, gastrointestinal and central nervous system complications, and considerable eosinophilia. The ESR remained low during these episodes with little clinical or histological evidence of vasculitis. Colonic biopsies obtained during the episode of colitis showed no evidence of granuloma formation or vasculitis. We therefore made a diagnosis of the hypereosinophilic syndrome.

Hydroxyurea has been used with success in cases of HES with poor prognostic features such as neurological and cardiac complications, and in those unresponsive to steroids. ${ }^{10}$ In our patient remission of 
the disease and the eosinophilia was achieved successfully with this drug, lending support to the suggestions that tissue damage in this syndrome is mediated by eosinophils.

\section{References}

1. Hardy, W.R. \& Anderson, R.E. The hypereosinophilic syndromes. Ann Intern Med 1968, 68: 1220-1229.

2. Chusid, M.J., Dale, D.C., Burton, C.W., West, M.D. \& Wolf, S.M. The hypereosinophilic syndrome. Analysis of fourteen cases with review of the literature. Medicine (Baltimore). 1975, 54: 1-27.

3. Spry, C.J.F., Davies, J., Tai, P.C., Olsen, E.G.J., Oakley, C.M. \& Goodwin, J.F. Clinical features of fifteen patients with the hypereosinophilic syndrome. $Q J \mathrm{Med}$ 1983, 205: 1-22.

4. Kasler, M.K. \& Sobin, H. Eosinophilic gastroenteritis. In Berk, J.E. (ed) Bockus Gastroenterology, 4th edition. Saunders, Philadelphia, 1985, Vol 2, chapter 82, pp. 1389-1395.

5. Suen, K.C. \& Burton, J.D. The spectrum of eosinophilic infiltration of the gastrointestinal tract and its relation-

\section{Acknowlegements}

We thank Dr A.G. Chappell for permission to report this case, Dr J. Morris and Dr E. Wilkins for performing the flexible sigmoidoscopies, and $\mathrm{Dr} A$. Rees for reporting the histology and providing copies of the colonic biopsies.

ship to other disorders of angiitis and granulomatosis. Hum Pathol 1979, 10: 31-43.

6. Cello, J.P. Eosinophilic gastroenteritis - A complex disease entity. Am J Med 1979, 67: 1097-1104.

7. Sherman, M.P. \& Cox, K.L. Neonatal eosinophilic colitis. J Pediatr 1982, 100: 587-589.

8. Churg, J. \& Strauss, L. Allergic granulomatosis, allergic angiitis and periarteritis nodosa. Am J Pathol 1951, 27: 277-301.

9. Lanham, J.G., Elkon, K.B., Pusey, C.D. \& Hughes, G.R. Systemic vasculitis with asthma and eosinophilia: A clinical approach to the Churg-Strauss Syndrome. Medicine (Baltimore) 1984, 63: 65-81.

10. Parillo, J.E., Fauci, A.S. \& Wolff, S.M. Therapy of the hypereosinophilic syndrome. Ann Intern Med 1978, 89: $167-172$. 